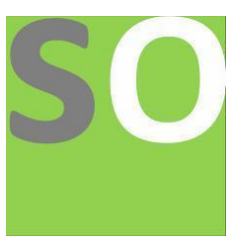

Article title: What to know in order to build a chess playing Al

Authors: Fabrizio Ginesi[1]

Affiliations: Liceo P. Frisi, Via sempione 21[1]

Orcid ids: 0000-0002-0489-6778[1]

Contact e-mail: fabrizio.ginesi@hotmail.it

License information: This work has been published open access under Creative Commons Attribution License http://creativecommons.org/licenses/by/4.0/, which permits unrestricted use, distribution, and reproduction in any medium, provided the original work is properly cited. Conditions, terms of use and publishing policy can be found at https://www.scienceopen.com/.

Preprint statement: This article is a preprint and has not been peer-reviewed, under consideration and submitted to ScienceOpen Preprints for open peer review.

DOI: 10.14293/S2199-1006.1.SOR-.PPPAZQN.v1

Preprint first posted online: 19 February 2021

Keywords: chess, Al, computer science, coding 
Fabrizio Ginesi

\section{What to know in order to build a chess playing $\mathrm{Al}$}

The results of my work: https://jsfiddle.net/fabrizio_11/gdm8c6fz/117/ Abstract:

Als that play chess have become more and more common on sites that before only let players battle against each other online, and their code is often much simpler than it may appear from an external eye. With some understanding of the formal language and experience not only is it possible to understand and recreate one, but even to correct some flaws this Als have, to make them more engaging for the player.

Index:

1 Requirements

1.1 Code knowledge

1.2 Libraries

1.3 Programs and sites used in the process

2 The objective

2.1 The planning

3 Time to code

4 The coding of the CSS board

4.1 The meaning of the main strings

5 The coding of the Javascript Al

5.1 The meaning of the main strings

6 Conclusions on the project

7 That's great, but where are we REALLY with the progress of Al?

8 References fundamental for the realization of the project

9 Other references to similar papers

\section{Requirements}

In this section we're going to quickly list the requirements that one should have when approaching a project like the one that l've just concluded. They are not binding in any way and surely the work you will do on this project also includes an enormous amount of self improvement and further development of your tech abilities, so don't be afraid to tackle this argument without being a professional programmer.

With that said, let's get to it!

\subsection{Code Knowledge}

The first thing we need to discuss while talking about a project like this is the pre-needed knowledge about coding that it requires. I will relate the discussion to my experience but I'm pretty certain that you can take it as the minimum requirements to meet:

-I am by no means a professional programmer, but I have previous experience in the field.

-I worked for a considerable amount of time for a Videogame developing 
company (Fantasy Fusion Studio) with Unity, which mainly uses $\mathrm{C}++$ as core language, and while for this project we are not going to use $\mathrm{C}$ it is extremely useful to be confident with a formal language such as this one, as you can learn the differences with the others (such as java or html) along the way, but at least you know how to orient yourself.

-l've worked on simulations for real life objects and physical behaviors such as rockets and missiles, which offered me a perspective into the usage of scripts and integrated models that use more coding languages at once, like the one we're creating.

\subsection{Libraries}

Luckily for everyone there are some javascript libraries that one can refer to while creating a project like this one, as coding from scratch the entire chess rules would be incredibly time consuming.

So, these are the one l've used:

- For chess rules: https://github.com/jhlywa/chess.js/

- For board composition: https://github.com/oakmac/chessboardjs/ As you can see they are not numerous, but they give a nice backbone to the entire project we can start building upon.

\subsection{Programs and sites used in the process}

Also for this part there's not that much to talk about, as I've only used a handful of programs and sites to complete the task.

For programs:

- Visual studio 2019 to see the project in its entirety

- Visual studio code to write the single strings as I find extremely useful its user-friendliness and autocode correction

For sites:

- https://github.com/ for the libraries

- https://stackoverflow.com/ for the inevitable code problems you'll incur

- https://jsfiddle.net/ to put the entire project on the web ready to be used and open source

\section{The objective}

I've recently got very interested in chess, and while I'm certainly not a master in it, l've found quite a pleasure in spending some free hours battling against an online opponent or an Al. Then, l've started questioning myself about those Al: "are they learning ? If so, how does that integrate with their preset difficulty? Does the number of consequent logical moves calculated vary from different difficulties or the only difference between a, let's say 1200 ELO (ranking chess points) and a $2500 \mathrm{ELO} A \mathrm{Al}$ is how they decide to act upon that consequent moves?" And so on and so on. So l've also started to question them with the games, as their code is inaccessible, sometimes by making moves they couldn't expect as they seemed unreasonable (and often were), sometimes by just extending a match even 
if the mate is calculated in one or two moves, or just about whatever floated in my mind while playing. The conclusion l've came across is that, at least while talking about the Als that are present in the main chess site right now (chess.com), they aren't learning at all, even the ones that don't declare a preset difficulty but declare an "adaptive" difficulty. They just follow the same move scheme every game, and even if sometimes it seems like it isn't the case, they all play in the same way, they just make better or worse moves based on the pre-set difficulty. This was honestly an enormous let down of my expectations, but at the same time it sparked in me the realization that maybe, through a thorough study of the subject I could develop something more...interesting for the player.

\subsection{The planning}

I was certain that a machine learning chess playing Al algorithm was already invented, and obviously it wasn't my intention to build a new one. I didn't and still don't have the abilities and knowledge required to do so. The only thing I wanted to do was to start from a pre-existing algorithm and develop my very own chess playing Al. So, while browsing the web l've stumbled upon the "minimax" algorithm, which seems to be the most favorable to chess programmers. Simply put, the minimax algorithm analyzes a "zero sum game" (a game where if player A makes a point player B makes minus one point, so the sum of points is always zero) and tries to maximes the earning while minimizing the losses. Obviously this algorithm can't be utilized as it is in chess, since the number of possible moves is circa $10^{\wedge} 120$, which is more than the millisecond we have till the inevitable heat death of the universe, so, no. What comes in play here is the "alpha-beta pruning" which is a supplementary research algorithm that, starting with the minimax, goes through the most favorable options of tree of consequences and "prunes" the non favorable ones without calculating them, and thus, without wasting enormous amount of time and resources.

An example of alpha beta data pruning on a minimax tree.

\section{Time to code}

In chapter 2 of the articole we have covered the main theoretical aspects one needs to know while writing an $\mathrm{Al}$, from now on instead we are going to analyze the proper code that lets us build the machine. I am not going to cover the entire code as I believe it might be a waste of time, as the target of this article are the ones that don't need an explanation for trivialities of the language but only it's main components. Also, the proper code on jsfiddle is going to be structured in such a way:

-The html site, on which l'm not gonna stop on since it is a very simple surface for the code.

-The CSS board, which recalls the library and let's us personalize the 
project.

-The JAVASCRIPT+JQUERY 2.2.4, which is the proper Al and bases itself on a series of mathematical principles that I'm gonna explain later in the article.

\section{The coding of the CSS board}

Let's put aside for a moment the discussion about the Al and let's analyze another fundamental part of any chess program: the board.

In fact, unlike what you might expect if you're not used to the usage of libraries in CSS and HTML, the fact that we have recalled a library for the chessboard is not sufficient for the board to exist on our site. It's like having all the right assets to build a house but not having the blueprint for it. But rest assured, this part is without a doubt the most easy as it only involves a certain degree of formal understanding of coding and some abilities in the decorative department to choose the right colors and patterns.

\subsection{The meaning of the main strings}

I won't paste the entirety of the CSS code on this paper as it would be a waste of precious space (you can find it entirely on the JSfiddle site).

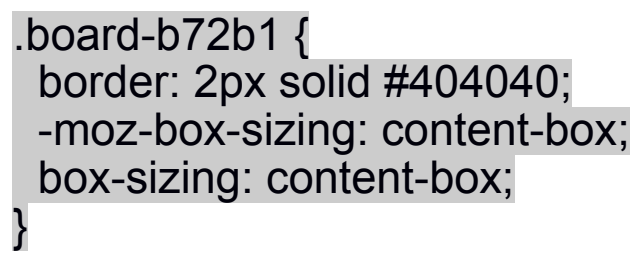

These are some generic infos on the chess box that are required for its existence.

\section{.square-55d63 \{}

float: left;

position: relative;

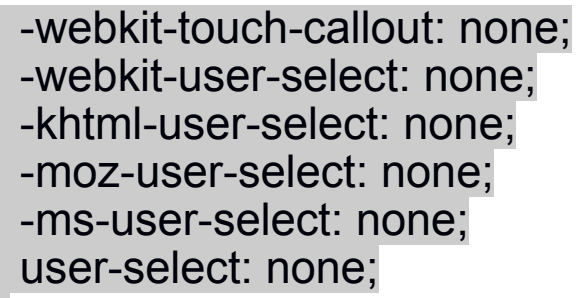

The infos for the squares of the board, as you can see they obviously must be made independent from the user selection or interaction with them. ( These are valid for both the white and the dark ones, the infos for the 
single ones are directly beneath these strings and are purely esthetical).

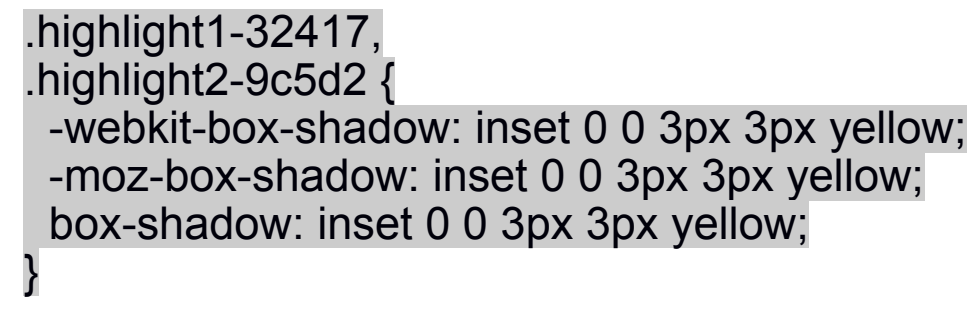

These strings are not directly related to the chess board as they are related to a little box that I wanted to insert in the program in order to ease the usage of the site for the user. As you can see it simply creates a yellow box around the moving piece, which is something I thought might come in handy for the player.

\section{The coding of the Al}

Now we approach the real deal of this paper, and while you recall the basic theoretical infoes l've given you on the subject, I hope I can properly explain how those meet actual practical realization. So, as we've seen the entirety of the chess algorithm bases itself on the necessity to gain the most with the least of the losses, which means that given a certain board, the Al will analyze the pieces that are currently movable from its side and decide based on the possible moves, that are translated in a series of and + values, which is the most valuable.

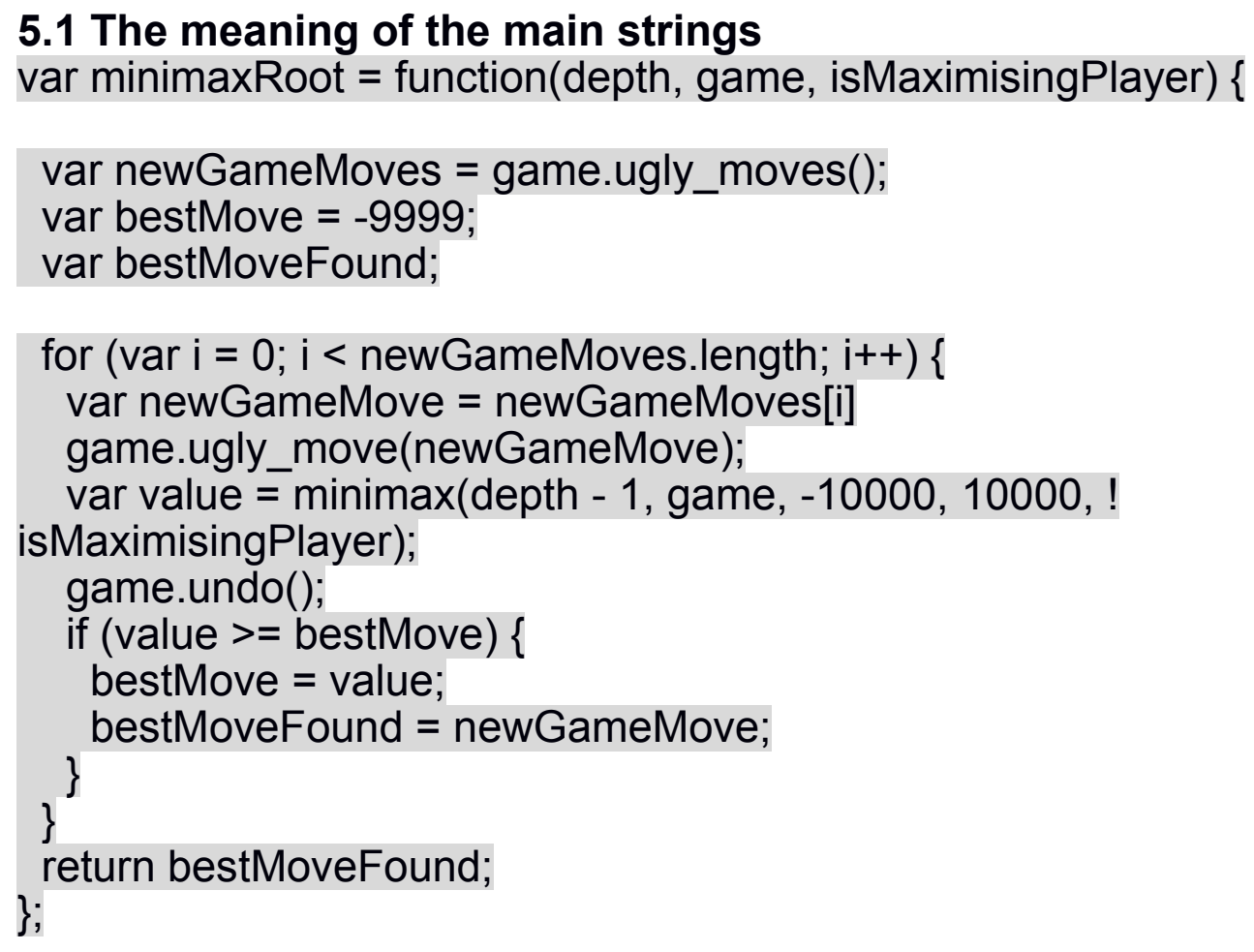


I believe that the strings above are the most crucial to understand the minimax algorithm works. As you can see it is declared that the bestMove has an astoundingly low value, since it is the lowest it can reach without being completely a loss, which is represented by the depth -1 with -10000 , but in case the Al finds a value that in the current situation is better than the -9999 move, it will be substituted to the bestMove and played (clearly the higher the better it is). You can also see that the root on which the entire minimax algorithm bases itself depends on the depth, or the difficulty, on which the game was pre-set. This is the fundamental change between this algorithm and the chess.com one, because while the latter just chooses different values on the tree depending on what difficulty the user has chosen, in this case the preset data tree completely changes itself in its values with the four difficulties, and with that, always presents a challenge for any player, no matter whether he is a beginner or a grandmaster.

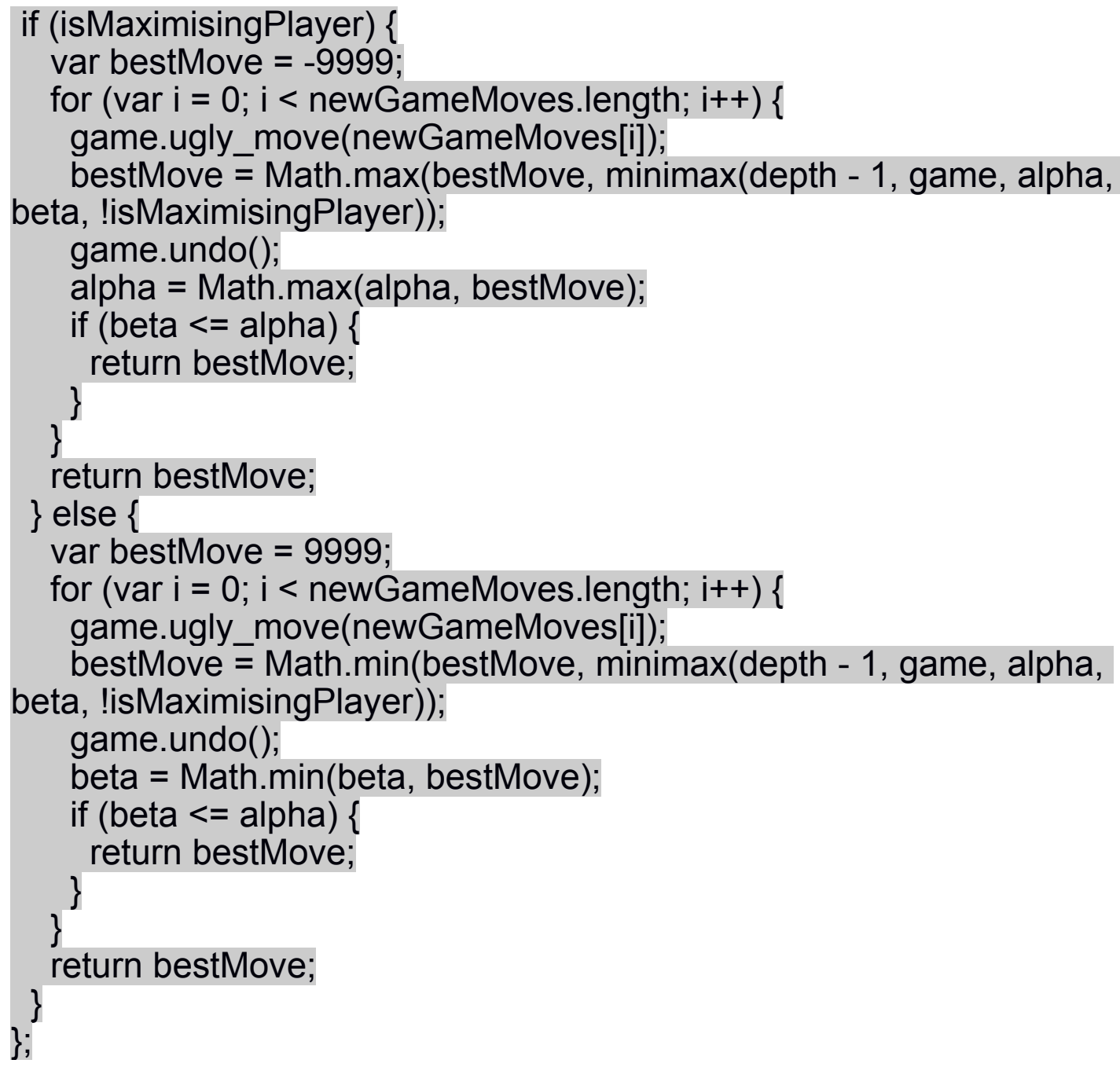

Now that we've covered how the minimax algorithm works in practicality 
let's also talk about the alpha beta pruning, which is used in the strings above. As you can see usage of this algorithm is fairly easy, if the Al is the Maximizing player, which simply put means that it recognizes that its winning, the BestMove starts at -9999 , and alpha is set as the mathematical max it can recover from there to maintain its advantage and if it is more than the other branch of minimax tree, which is beta, it is chosen. The same goes in case the Al is losing, but instead the BestMove is set at 9999 and the Al tries to calculate the move with which it can gain more points as possible to recover from the disadvantaged position. In this case it is calculated the mathematical minimum for beta as it is trying to achieve a minimal loss, that if compared to alpha is bigger, is discarded in favor of the latter.

\section{Conclusions on the project}

I've conducted this project alone for long, and since l've started it with one can only be described as a "tarnished knowledge" of Al, I can proudly say that l've learned a lot from it and it has given me an enormous amount of satisfaction to see it finally completed. I wanted to write this short paper to pass on a message, which simply put is, "if I did it, you can do it". We often feel scared to challenge ourselves with tasks like the one l've written about, but in order to mature and reach a better knowledge of the world, we need to take risks. Learn to make machines learn.

\section{That's great, but where are we REALLY with the progress of Al?} Now that we've concluded this rudimental first bot, we might ask ourselves: "but at what point are we really with the Al?" Well, much further. To put it in perspective the chess playing Al we've built here can compete with players up to an ELO score of 2200-2300, which is not even a grandmaster level (2500), while the strongest Al at the moment have long beaten the champions in the world and have an ELO of around 3200, which constrains them to play in a league of their own, without the presence of faulty humans! We have reached a point where to create a truly interesting experience for the player the Als have started to make faults "willingly" (more on that here https://www.wired.com/story/new-artificial-intelligencemistakes-purpose-chess/).

But even outside of the world of chess, Al is revolutionating every field it manages to touch, from sports by coaching, simulating games and broadcasting (https://medium.com/@mygreatlearning/how-is-ai-used-insports-cdfbdd97ad82) to graphic design and art with, for example, the new Adobe tool "sensei" which makes graphic design much easier and approachable (https://www.clickz.com/design-by-computers-how-ai-ischanging-the-graphic-design-industry/262191/)

Overall, $\mathrm{Al}$ is changing not only the world but also ourselves, by cutting short on the repeated tasks and letting us express our inner abilities freely, which is what ultimately makes us humans. 


\section{References fundamental for the realization of the project}

I wanna thank the following, without whom this project would have never been possible:

-Lauri Hartikka with her "A step by step guide to building a simple chess Al" https://www.freecodecamp.org/news/simple-chess-ai-step-by-step1d55a9266977/

-"Chess programming wiki", a true Bible for any of my Al related issues https://www.chessprogramming.org/Artificial_Intelligence

-Marissa Eppes "The Minimax algorithm explained" https://towardsdatascience.com/how-a-chess-playing-computer-thinksabout-its-next-move-8f028bd0e7b1

-"Theory of Games and Economic behavior" by John von Neumann and Oskar Morgenstern, a fundamental basis for anyone who might be interested on the matter.

\section{Other references to similar papers}

Other similar articles to the one you've just read can be find here:

"Chess as the Drosophila of Al" by J.McCharty https://link.springer.com/chapter/10.1007/978-1-4613-9080-0 14

"IBM's Chess Players: On Al and Its Supplements" by Brian P. Bloomfield and Theo Vurdubakis https://www.tandfonline.com/doi/abs/10.1080/01972240701883922

"Computers, chess, and cognition" by T.Anthony Marsland and Jonathan Schaeffer https://link.springer.com/book/10.1007/978-1-4613-9080-0

"Aligning Superhuman Al with Human Behavior: Chess as a Model System" by Reid Mcllroy-Young Siddhartha Sen,Jon Kleinberg and Ashton Anderson

https://dl.acm.org/doi/abs/10.1145/3394486.3403219?

casa token=NdQ5UL5BdgQAAAAA

\%3Acz1iX83bfGQ10pW pfXoR5 sRWObKXiQ0-

f8nEGZBpO_JSCLRDMas6jS6Kj4_qCsASGshqi2DPf

"Artificial Intelligence: Chess match of the century" by Demis Hassabis https://www.nature.com/articles/544413a

"On the Creation of a Chess-Al-Inspired Problem-Specific Optimizer 
for the Pseudo Two-Dimensional Battery Model Using Neural

Networks" by Neal Dawson-Elli, Suryanarayana Kolluri, Kishalay Mitra and Venkat R. Subramanian

https://iopscience.iop.org/article/10.1149/2.1261904jes/meta

"Adaptive Pattern-Oriented Chess" by R. Levinson and R. Snyder

https://www.sciencedirect.com/science/article/pii/B9781558602007500210

"The impact of chess research on cognitive science" by Neil Charness https://link.springer.com/article/10.1007/BF01359217

"The role of chess in artificial intelligence research" by R. Levinson, F. Hsu, J. Scheffer, T. Marsland, D. Wilkins

https://pdfs.semanticscholar.org/fac0/dd639b47514c89efa0393c3b6c29a1 d408e6.pdf

"Deep Blue's contribution to Al by Monty Newborn"

https://link.springer.com/article/10.1023/A:1018939819265 